\title{
Origin of resistivity contrast in interfacial phase-change memory: The crucial role of $\mathrm{Ge} / \mathrm{Sb}$ intermixing
}

\author{
Yuta Saito, ${ }^{1,2, a)}$ Alexander V. Kolobov, ${ }^{1,3}$ Paul Fons, ${ }^{1}$ Kirill V. Mitrofanov, ${ }^{1}$ Kotaro Makino, ${ }^{1}$ \\ Junji Tominaga, ${ }^{1}$ and John Robertson ${ }^{2}$
}

${ }^{1}$ National Institute of Advanced Industrial Science and Technology (AIST), Tsukuba 305-8565, Japan

${ }^{2}$ Department of Engineering, University of Cambridge, Cambridge CB3 OFA, United Kingdom

${ }^{3}$ Faculty of Physics, Herzen State Pedagogical University, St Petersburg 191186, Russia

a)yuta-saito@aist.go.jp

\begin{abstract}
Phase-change memories based on reversible amorphous-crystal transformations in pseudobinary $\mathrm{GeTe}-\mathrm{Sb}_{2} \mathrm{Te}_{3}$ alloys are one of the most promising non-volatile memory technologies. The recently proposed superlattice-based memory, or interfacial phase change memory (iPCM), is characterized by significantly faster switching, lower energy consumption and better endurance. The switching mechanism in iPCM, where both the SET and RESET states are crystalline, is still contentious. Here, using the $a b$ initio density functional theory simulations, a conceptually new switching mechanism for iPCM is derived, which is based on the change in potential landscape of the band-gap, associated with local deviations from the pseudo-binary stoichiometry across the van der Waals gaps, and the associated shift of the Fermi level. The crucial role in this process belongs to $\mathrm{Ge} / \mathrm{Sb}$ intermixing on the cation planes of iPCM. These findings offer a comprehensive understanding of the switching mechanisms in iPCM and are an essential step forward to the insightful development of phase-change memory technology.
\end{abstract}


Phase change random access memory (PCRAM) is one of the most promising candidates for the next generation non-volatile memory with phase change materials forming a key component of the recently commercialized 3D XPoint memory technology where sophisticated three-dimensional integration of chalcogenide-based memory and selector components enables much faster writing speeds than Flash and denser capacity than dynamic random access memory (DRAM). ${ }^{1,2}$ The development of phase-change materials dates back over three decades, with the most studied materials being Ge-Sb-Te (GST) ternary alloys on the GeTe$\mathrm{Sb}_{2} \mathrm{Te}_{3}$ pseudobinary tie-line, and which found great success in optical disc devices. ${ }^{3}$ On the other hand, as optical disc and non-volatile memories clearly have very different requirements, the required material properties also differ. Using the same material, GST alloys, for PCRAM memories does not in itself solve the various difficulties in developing non-volatile memories.

A major breakthrough in PCRAM materials was the development of interfacial phase change memory (iPCM) inspired by the Ge-atom flip-flop model. ${ }^{4-8}$ In iPCM, the two binary end compounds of the GeTe-Sb $\mathrm{Te}_{3}$ pseudobinary tie line, GeTe and $\mathrm{Sb}_{2} \mathrm{Te}_{3}$, are alternately stacked to form an atomically aligned superlattice structure with van der Waals (vdW) gaps separating covalently bonded blocks. Devices based upon iPCM showed excellent performance such as ultra-low power consumption, faster switching speeds, and longer endurance than conventional alloy-type PCRAM. ${ }^{4,9}$

After the development of chalcogenide superlattices and their devices based on $\mathrm{GeTe} / \mathrm{Sb}_{2} \mathrm{Te}_{3}$ superlattices fabricated from sputter-deposited films, ${ }^{4,10-13}$ several specific switching models were proposed based on the idea of bi-layer switching within GeTe blocks. ${ }^{14-20}$ In particular switching between inverted Petrov and Ferro structures (Figure 1) was proposed based on the arguments that the relative stability of these two phases change with temperature. ${ }^{14}$ 
At the same time, superlattices have also been fabricated in a single crystal form using molecular beam epitaxy (MBE) ${ }^{21-24}$ and pulsed laser deposition (PLD) $)^{25}$ and comprehensive transmission electron microscopy (TEM) experiments were carried out on such samples. ${ }^{26-31}$ These measurements demonstrated that the (as-grown) structures were different from the original intuitive models proposed for the iPCM SET and RESET states. In particular, it was found that (1) in as-grown samples, Ge atoms are predominantly located towards the core of covalently bonded blocks rather than between $\mathrm{Sb}_{2} \mathrm{Te}_{3}$ quintuple layers while (2) $\mathrm{Sb}$ atoms are mainly located closer to vdW gaps. ${ }^{26}$ It was additionally shown that (3) Ge/Sb atoms intermix, so that no pure Ge or Sb planes exist. ${ }^{30}$ Furthermore, despite a very precise growth technique such as MBE, (4) the number of atomic planes in covalently bonded blocks in Ge-Sb-Te varies throughout the sample thickness, where covalently bonded blocks with (various) odd numbers of planes co-exist. ${ }^{32}$ At the same time, we note that in pure $\mathrm{Sb}_{2} \mathrm{Te}_{3}$, only 5-layer blocks are observed (Fig. 1). Also, (5) there is clear asymmetry in the $\mathrm{Ge} / \mathrm{Sb}$ concentration ratio on different sides of vdW gap, possibly associated with the SL growth direction ${ }^{33}$ and (6) dynamical vdW gap reconfiguration consisting of a bilayer switching across the gap is a lowenergy process. ${ }^{34}$

These results called for a reconsideration of the phase-change mechanism in iPCM, which is critical in order to launch industrial development of iPCM-based devices. In this Letter, we shed light on the switching mechanism on $\mathrm{GeTe} / \mathrm{Sb}_{2} \mathrm{Te}_{3}$ superlattices and the origin of the high resistance contrast by $a b$ initio density functional theory (DFT) simulations using structural models based on the latest experimental findings. In particular, we demonstrate that $\mathrm{Ge} / \mathrm{Sb}$ intermixing, which at the first glance seemed to be a detrimental factor, is in fact a feature crucial for iPCM switching. 
The simulations of bi-layer switching were performed using the density functional code CASTEP $^{35}$ with the GGA-PBE ${ }^{36}$ exchange potential, ultrasoft pseudopotentials, and the DFTD2 dispersion correction (Grimme) to account for vdW intereaction. ${ }^{37}$ A plane wave cutoff energy of $230 \mathrm{eV}$ was used with a $2 \times 2 \times 1$ Monkhorst-Pack grid. The electronic density of states (DOS) was calculated using the all-electron LAPW code WIEN2k ${ }^{38}$ with a Monkhorst-Pack grid of $10 \times 10 \times 10$, an $R_{M T} K_{M A X}$ of 7, spin-orbit coupling, and the modified Becke-Johnson LDA functional. ${ }^{39}$

First, we consider how bi-layer switching across vdW gaps, reported experimentally by various groups and named dynamic gap reconfiguration, ${ }^{31-33}$ affects the electronic structure. Figure 2(a) shows a schematic of the proposed bi-layer switching process $(9+9 \rightarrow 7+11$, where numbers indicate the number of atomic layers). We start with GeTe bilayer switching from ...Te-Sb-Te-Ge-Te-Sb-Te-Ge-Te...Te-Sb-Te-Ge-Te-Sb-Ge-Te... (9+9) to ...Te-Sb-Te-GeTe-Sb-Te...Te-Ge-Te-Sb-Te-Ge-Te-Sb-Te Ge-Te... (7+11) structures (Fig. 2(a)). In this case the switching is between local GST225+GST225 and GST124+GST326, i.e. all local compositions lie on the pseudobinary tie-line. It should be noted that the atomic ordering on GST225 is neither pure Kooi nor Petrov structure, but there is an asymmetry of cation layers in order to realize GeTe bilayer switching. Therefore, this structure is designated GST225_K_P as the bottom side has the Sb layer close to the vdW gap (Kooi-like) and the top side is Ge (Petrov-type). The electronic DOS corresponding to these atomic blocks are shown in Fig. 2(b). One can see that the GeTe switching has a negligible effect on the electronic structure, i.e. the semiconducting gap is preserved and the Fermi energy is always the middle of the gap. It should be also noted that the Fermi energy is located at the middle of the gap also for the pseudobinary symmetric Petrov and Kooi phases, i.e. pseudobinary materials are always intrinsic regardless of the stacking order. 
We proceed with the opposite case of pure SbTe bi-layer switching from one GST225_K block to another. Such switching results in the formation of GST214 and GST236 (Fig. 2(c)), i.e. the compositions that do not lie along the GeTe-Sb $\mathrm{Te}_{3}$ tie-line, viz., GST214 is Ge-rich while GST236 is Sb-rich. The electronic DOS of these two structures are shown in Fig. 2(d). Both models have a narrow band gap of about $0.2-0.3 \mathrm{eV}$ but, intriguingly, the Fermi energy is located in the valence band and in the conduction band for the GST214 and GST236 compositions, respectively, suggesting that GST214 is p-type and GST236 is an $n$-type semiconductor. This can be understood using an electron counting model similar to that of Chen et al.. ${ }^{40,41}$ In their model, the valence $p$-electrons were considered, and since $\mathrm{Ge}$ and $\mathrm{Sb}$ can be treated as cations and Te as an anion, the sign of electron counting is opposite for $\mathrm{Ge} / \mathrm{Sb}$ and Te. For GST225, the electron balance of the unit cell is as follows: $2 \times 2+3 \times 2-2 \times 5=0$ giving a net charge of zero. On the other hand, the electron balance for GST214 is $2 \times 2+3 \times 1-2 \times 4=-1$, while that for GST236 is $2 \times 2+3 \times 3-2 \times 6=+1$. This implies that GST214 is electron deficient while GST236 has an electron excess, resulting in the $p$-type and $n$-type tendencies of GST214 and GST236, respectively.

Next, to reflect experimental findings we performed simulation of bi-layer switching for a model that includes $\mathrm{Ge} / \mathrm{Sb}$ intermixing. The process is schematically illustrated in Figure 3. and it was found that the energy barrier for each switching process requires low energy so that in principle the bi-layer switching can take place between any combinations of vdW blocks leading to many additional degrees of freedom for the formation of different vdW blocks in actual superlattice structures. ${ }^{34,40}$

Now, based on the obtained electronic structures for GeTe and SbTe bilayer switching as well as structural dynamics, we extend our idea into a more general case. Figure 4 shows a GeSb-Te ternary phase diagram including some off-tie-line compositions. The well-known GeTe- 
$\mathrm{Sb}_{2} \mathrm{Te}_{3}$ pseudobinary tie-line has been drawn as a red line, and selected ternary compounds have been indicated alongside. The pink dashed lines represent compositions with different numbers of atomic layers in the vdW blocks. Since the number of atomic layers is determined by the number of Te layers, these lines also indicate constant Te composition. In this more general case, any composition in principle can be realized. For example, the outermost cation layer may be composed of half $\mathrm{Ge}$ and half $\mathrm{Sb}$ atoms. In this case, the outermost bi-layer can be represented as $\mathrm{Ge}_{0.5} \mathrm{Sb}_{0.5} \mathrm{Te}$ (instead of pure $\mathrm{SbTe}$ ), and consequently, after switching the final compositions of the two constituent blocks will become $\mathrm{Ge}_{1.5} \mathrm{Sb}_{1.5} \mathrm{Te}_{4}$ and $\mathrm{Ge}_{2.5} \mathrm{Sb}_{2.5} \mathrm{Te}_{6}$. By analogy with the electron counting model as well as the DOS shown in Fig. 2, it can be easily understood that the former composition is electron deficient ( $p$-type) while the latter is $n$-type (with the type depending on the which side of the pseudobinary tie-line a given composition lies). Variation of composition with respect to the pseudobinary tie-line is thus an efficient way to control the Fermi level position. The importance of the stoichiometric compositions was also discussed based on DFT. ${ }^{42}$ We also note that the impact of the stoichiometric compositions on iPCM structures was reported to be critical to realize reliable devices. $^{43}$

Based on these considerations, the origin of electrical contrast in PCM can be deduced. Before doing so, it should be noted that crystalline GST materials are usually degenerate $p$ type semiconductors due to negatively charged defects arising from $\mathrm{Ge}$ vacancies $\left(\mathrm{V}_{\mathrm{Ge}}{ }^{2-}\right.$ $\left.+2 \mathrm{~h}^{+}\right) .{ }^{44,45}$ Therefore, in actual films, the Fermi energy is located at the top of the valence band. A schematic DOS of standard "on-line" compounds such as GST124 or GST225 is shown in Figure 5(a) top. If an electron deficient compound is formed following bilayer switching, such as GST214, this leads to additional hole doping, driving the Fermi energy even deeper into the valence band (Figure 5(a) center). In contrast, an electron surplus compound results in extra electrons that compensate the pre-existing holes shifting the Fermi energy towards mid-gap as 
shown in Fig. 5(a) bottom, resulting in a higher resistivity. The spatial non-uniformity of the local stoichiometry with respect to the pseudobinary tie-line is reminiscent of the band structure of a disordered semiconductor (Fig. 5(b)), where the valence and conduction band edges fluctuate in order to maintain a constant Fermi energy throughout the sample, resulting in the creation of localized "pools of holes" ${ }^{46,47}$ As a consequence, the resistivity of the system increases. $^{46}$

In what follows, based on the reported results, we propose a comprehensive switching model for iPCM that resolves contradictions existing in literature. We remind the readers that in conventional PCRAM, application of a SET pulse results in the crystallization of the disordered phase, while a RESET pulse raises the local temperature above the melting point leading to the melt-quenching of the material to form the disordered phase. By analogy, since the SET state in iPCM is the energetically most stable low resistance state, vdW blocks with standard compositions along the tie-line such as GST124 (7-layers) or GST225 (9-layers) are expected to be dominant. This is consistent with the statistical TEM studies ${ }^{32}$ which found that after annealing between $300-400^{\circ} \mathrm{C}$, the distribution of vdW blocks was centered upon 7- and 9vdW layer blocks (Fig. 5(b) top, (c) left).

On the other hand, once a RESET pulse is applied to an iPCM device, the superlattice may re-arrange due to dynamic bi-layer switching of $\mathrm{Ge} / \mathrm{Sb}$-containing layers. Because of the shorter duration of the RESET pulse, the off-line composition blocks are "quenched" after the termination of the pulse. This leads to a wider distribution of $E_{\mathrm{V}}-\mathrm{E}_{\mathrm{F}}$ values, and the creation of local regions in which the Fermi energy is located inside the band gap resulting in a high resistance state (Figure 5(b) bottom, (c) right). It is these high-resistive blocks that determine the total resistance of the device as the current flows vertically through the vdW blocks. 
Furthermore, the polarity of the electric field may be also important as electric field-induced switching has also been proposed. ${ }^{48}$

The microscopic bi-layer switching scenario may be as follows. First, we notice that in the hexagonal structure (Figure 6(a)) the arrangement of Te atoms around the vdW gap is such that there are numerous tetrahedral (and only tetrahedral) vacancies formed by Te atoms. Second, the structure around the gap is asymmetric (as evidenced by TEM images ${ }^{26}$ ), i.e. on one (bottom in the Figure) side of the vdW gap there are more Ge atoms than at the opposite (top in the Figure) side. In such a case, a temperature increase due to an applied RESET pulse may drive some of the Ge atoms from the lower layer adjacent to the gap into the existing tetrahedral vacancies, because Ge atoms usually prefer tetrahedral coordination (Fig. 6(b)). We further note that at elevated temperatures, when the mobility of atoms is increased, the direction of the applied electric field may also play a role. Because $\mathrm{Ge} / \mathrm{Sb}$ and Te have opposite charges, for the electric field direction shown in the Figure, Ge/Sb atoms above the vdW gap will be pushed upward, i.e. away from the gap, while those located below the gap will be pushed into the vdW gap. Once the number of switched Ge atoms approaches a critical value (Fig. 6(c)), Te layers across the vdW gap slide (Fig. 6(d)) and the energetically more favorable octahedrally bonded configuration of $\mathrm{Ge}$ is established thanks to the collective effect of resonant ${ }^{49}$ (recently also called metavalent ${ }^{50}$ ) bonding. We note here that this is the reverse process of what has been proposed for the cubic-to-hexagonal transformation. ${ }^{51}$ This layer slide results in the transformation of the originally tetrahedral vacancy sites in the gap into octahedral vacancies, into which $\mathrm{Sb}$ atoms can subsequently switch (Fig. 6(e)), leading to the formation of a stacking fault and consequent movement of the vdW gap (two atomic planes down in the Figure), which, in turn, results in a shift of the Fermi level. Obviously, the above description is just a scheme, and these processes are not isolated and the structural transformation is a continuous single process. 
Based on the model proposed in Fig. 5, several points will allow further optimization of the switching and electrical properties of chalcogenide superlattice memory. One potential avenue for improvement is doping, a process that is also key to the Si semiconductor industry. For instance, doping with $\mathrm{Bi}$ could be used to tune the electronic structure as $\mathrm{Bi}_{2} \mathrm{Te}_{3}$ tends to be an $n$-type semiconductor due to point defects. Indeed, controlled Bi doping has been reported to lead to intrinsic behavior in $\left(\mathrm{Sb}_{1-\mathrm{x}} \mathrm{Bi}_{\mathrm{x}}\right)_{2} \mathrm{Te}_{3}$ with respect to the study on topological insulators. ${ }^{52}$ Some attempts to replace Ge by different elements have also been reported; tuning the composition has an even greater possibility of further improving iPCM performance. ${ }^{53,54}$

In conclusion, we have explored the structural dynamics and electronic structural changes in iPCM structures. The ease of bi-layer switching enables reversible structural changes driven by thermal and possibly also electric field effect of the applied external electrical stimuli. The existence of locally non-pseudo-binary metastable compositions across vdW gaps gives a degree of freedom for local doping that results in the shift of the Fermi level position and the concomitant shift of the Fermi level resulting in the experimentally observed resistance change. We also note that the presence of both $\mathrm{Ge}$ and $\mathrm{Sb}$ atoms on cation planes adjacent to vdW gaps, i.e. Ge/Sb intermixing, is crucial for the iPCM switching. Namely, the Ge atoms, due to their ability to acquire either tetrahedral or octahedral bonding geometry, facilitate the switching process while the switching of Sb breaks the electron balance resulting in the resistance change. These observations offer a comprehensive understanding of the switching mechanism in interfacial phase change memory and will be essential for the further development and commercialization of phase-change memory.

We expect that the ideas developed in this work will stimulate further theoretical and experimental studies. The TEM results obtained to date, while being a great step forward in understanding the iPCM structure, were performed on as-grown samples in thermal 
equilibrium and cannot be unambiguously associated with either the SET or RESET states. Indeed, in conventional PCM, the most stable phase is hexagonal but the reversible phasechange process occurs between the melt-quenched amorphous and metastable cubic phases. It is thus crucial, in order to understand the switching mechanism in detail, to perform direct observations using cross-sectional atomic scale resolution TEM imaging of iPCM devices switched between both resistance states over several cycles, which is currently a very challenging task.

Also, ab initio molecular dynamics simulations may be useful to understand the reversible switching between two different atomic layer configurations. In particular, structural simulations under the influence of an electric field may provide important insights. The use of the neural network approach, which allows one to handle large clusters using DFT, ${ }^{55}$ is especially appealing.

\section{ACKNOWLEDGMENT}

This study was supported by JST CREST No. JPMJCR14F1 and KAKENHI JP16K04896 and 18K14306. 


\section{REFERENCES}

(1) M. Wuttig and N. Yamada, Nat. Mater. 6, 824 (2007).

(2) P. Noé, C. Vallée, F. Hippert, F. Fillot, and J.-Y. Raty, Semicond. Sci. Technol. 33, 013002 (2018).

(3) N. Yamada, E. Ohno, K. Nishiuchi, N. Akahira, and M. Takao, J. Appl. Phys. 69, 2849 (1991).

(4) A. V. Kolobov, P. Fons, A. I. Frenkel, A. L. Ankudinov, J. Tominaga, and T. Uruga, Nat. Mater. 3, 703 (2004).

(5) J. Tominaga, P. Fons, A. Kolobov, T. Shima, T. C. Chong, R. Zhao, H. K. Lee, and L. Shi, Jap. J. Appl. Phys. 47, 5763 (2008).

(6) R. E. Simpson, P. Fons, A. V. Kolobov, T. Fukaya, M. Krbal, T. Yagi, and J. Tominaga, Nat. Nanotech. 6, 501 (2011).

(7) B. Huang and J. Robertson, Phys. Rev. B, 81, 081204 (2010).

(8) J. Tominaga, Phys. Status Solidi RRL, DOI: 10.1002/pssr.201800539 (2018).

(9) X. Zhou, J. K. Behera, S. Lv, L. Wu, Z. Song, and R. E. Simpson, Nano Futures, 1, 025003 (2017).

(10) R. E. Simpson, P. Fons, A. V. Kolobov, M. Krbal, and J. Tominaga, Appl. Phys. Lett. 100, 021911 (2012).

(11) M. Tai, M. Kinoshita, T. Ohyanagi, T. Morikawa, K. Akita, and N. Takaura, Jap. J. Appl. Phys. 54, 05ED01 (2015).

(12) Y. Saito, P. Fons, A. V. Kolobov, and J. Tominaga, Phys. Status Solidi (b) 252, 2151 (2015).

(13) Y. Saito, P. Fons, L. Bolotov, N. Miyata, A. V. Kolobov, and J. Tominaga, AIP Adv. 6, 045220 (2016).

(14) J. Tominaga, A. V. Kolobov, P. Fons, T. Nakano, and S. Murakami, Adv. Mat. Interfaces, 1, 1300027 (2014).

(15) N. Takaura, T. Ohyanagi, M. Tai, M. Kinoshita, K. Akita, T. Morikawa, H. Shirakawa, M. Araidai, K. Shiraishi, Y. Saito, and J. Tominaga, IEDM Tech. Dig. 29.2.1 (2014).

(16) X. Yu and J. Robertson, Sci. Rep. 5, 12612 (2015).

(17) J. Kalikka, X. Zhou, J. Behera, G. Nannicini, and R. E. Simpson, Nanoscale, 8, 18212 (2016).

(18) Y.-S. Song, J. Kim, and S.-H. Jhi, J. Appl. Phys. 121, 095102 (2017).

(19) H. Nakamura, I. Rungger, S. Sanvito, N. Inoue, J. Tominaga, and Y. Asai, Nanoscale, 9. 9386 (2017). 
(20) H. Shirakawa, M. Araidai, and K. Shiraishi, Jap. J. Appl. Phys. 57, 04 FE08 (2018).

(21) J. E. Boschker, E. Tisbi, E. Placidi, J. Momand, A. Redaelli, B. J. Kooi, F. Arciprete, and R. Calarco, AIP Adv. 7, 015106 (2017).

(22) J. E. Boschker and R. Calarco, Adv. Phys. X, 2, 675 (2017).

(23) S. Cecchi, R. N. Wang, E. Zallo, and R. Calarco, Nanosci. Nanotechnol. Lett. 9, 1114 (2017).

(24) R. Wang, F. R. Lange, S. Cecchi, M. Hanke, M. Wuttig, and R. Calarco, Adv. Func. Mater. 28, 1705901 (2018).

(25) I. Hilmi, A. Lotnyk, J. W. Gerlach, P. Schumacher, and B. Rauschenbach, Mater. Des. 115, 138 (2017).

(26) J. Momand, R.Wang, J. E. Boschker, M. A. Verheijen, R. Calarco, and B. J. Kooi, Nanoscale, 7, 19136 (2015).

(27) J. Momand, F. R. L. Lange, R. Wang, J. E. Boschker, M. A. Verheijen, R. Calarco, M. Wuttig, and B. J. Kooi, J. Mater. Res. 31, 3115 (2016).

(28) B. Casarin, A. Caretta, J. Momand, B. J. Kooi, M. A. Verheijen, V. Bragaglia, R. Calarco, M. Chukalina, X. Yu, J. Robertson, F. R. L. Lange, M. Wuttig, A. Redaelli, E. Varesi, F. Parmigiani, and M. Malvestuto, Sci. Rep. 6, 22353 (2016).

(29) R. Wang, V. Bragaglia, J. E. Boschker, and R. Calarco, Cryst. Growth Des. 16, 3596 (2016).

(30) A. Lotnyk, I. Hilmi, U. Ross, and B. Rauschenbach, Nano Res. 11, 1676 (2018).

(31) A. Lotnyk, U. Ross, T. Dankwort, I. Hilmi, L. Kienle, and B. Rauschenbach, Acta Mater. 141, 92 (2017).

(32) J. Momand, R. Wang, J. E. Boschker, M. A. Verheijen, R. Calarco, and B. J. Kooi, Nanoscale, 9, 8774 (2017).

(33) A. V. Kolobov, P. Fons, Y. Saito, and J. Tominaga, ACS Omega 2, 6223 (2017).

(34) X. Yu and J. Robertson, Sci. Rep. 6, 37325 (2016).

(35) S. J. Clark, M. D. Segall, C. J. Pickard, P. J. Hasnip, M. I. J. Probert, K. Refson, and M. C. Payne, Z. Kristallogr. 220, 567 (2005).

(36) J. P. Perdew, K. Burke, and M. Ernzerhof, Phys. Rev. Lett. 77, 3865 (1996).

(37) S. Grimme, J. Comput. Chem. 25, 1463 (2004).

(38) K. Schwarz and P. Blaha, Comp. Mater. Sci. 28, 259 (2003).

(39) F. Tran and P. Blaha, Phys. Rev. Lett. 102, 226401 (2009).

(40) N. K. Chen, X. B. Li, X. P. Wang, S. Y. Xie, W. Q. Tian, S. Zhang, and H. B. Sun, IEEE Trans. Nanotechnol. 17, 140 (2018). 
(41) X.-B. Li, N.-K. Chen, X.-P. Wang, and H.-B. Sun, Adv. Funct. Mater. DOI:10.1002/adfm.201803380 (2018).

(42) S. Caravati, M. Bernasconi, T. D. Kühne, M. Krack, and M. Parrinello, J. Phys.: Condens. Matter, 21, 255501 (2009).

(43) P. Kowalczyk, F. Hippert, N. Bernier, C. Mocuta, C. Sabbione, W. Batista-Pessoa, and P. Noé, Small, 14, 1704514 (2018).

(44) A. H. Edwards, A. C. Pineda, P. A. Schultz, M. G. Martin, A. P. Thompson, H. P. Hjalmarson, and C. J. Umrigar, Phys. Rev. B, 73, 045210 (2006).

(45) W. Zhang, A. Thiess, P. Zalden, R. Zeller, P. H. Dederichs, J.-Y. Raty, M. Wuttig, S. Blügel, and R. Mazzarello, Nat. Mater. 11, 952 (2012).

(46) F. Fritzsche, J. Non-Cryst. Sol. 6, 49 (1971).

(47) T. Siegrist, P. Jost, H. Volker, M. Woda, P. Merkelbach, C. Schlockermann, and M. Wuttig, Nat. Mater. 10, 202 (2011).

(48) T. Shintani and T. Saiki, Appl. Phys. Exp. 6, 111401( 2013).

(49) K. Shportko, S. Kremers, M. Woda, D. Lencer, J. Robertson, and M. Wuttig, Nat. Mater. 7, 653 (2008).

(50) M. Zhu, O. Cojocaru-Mirédin, A. M. Mio, J. Keutgen, M. Küpers, Y. Yu, J.-Y. Cho, R. Dronskowski, and M. Wuttig, Adv. Mat. 30, 1706735 (2018).

(51) B. Zhang, W. Zhang, Z. Shen, Y. Chen, J. Li, S. Zhang, Z. Zhang, M. Wuttig, R. Mazzarello, E. Ma, and X. Han, Appl. Phys. Lett. 108, 191902 (2016).

(52) J. Zhang, C.-Z. Chang, Z. Zhang, J. Wen, X. Feng, K. Li, M. Liu, K. He, L. Wang, X. Chen, Q.-K. Xue, X. Ma, and Y. Wang, Nat. Commun. 2, 574 (2011).

(53) S. Soeya, T. Shintani, T. Odaka, R. Kondou, and J. Tominaga, Appl. Phys. Lett. 103, 053103 (2013).

(54) Y. Saito, J. Tominaga, P. Fons, A. V. Kolobov, and T. Nakano, Phys. Status Solidi (RRL), 8, 302 (2014).

(55) G. C. Sosso, G. Miceli, S. Caravati, F. Giberti, J. Behler, and M. Bernasconi, J. Phys. Chem. Lett. 4, 4241 (2013). 


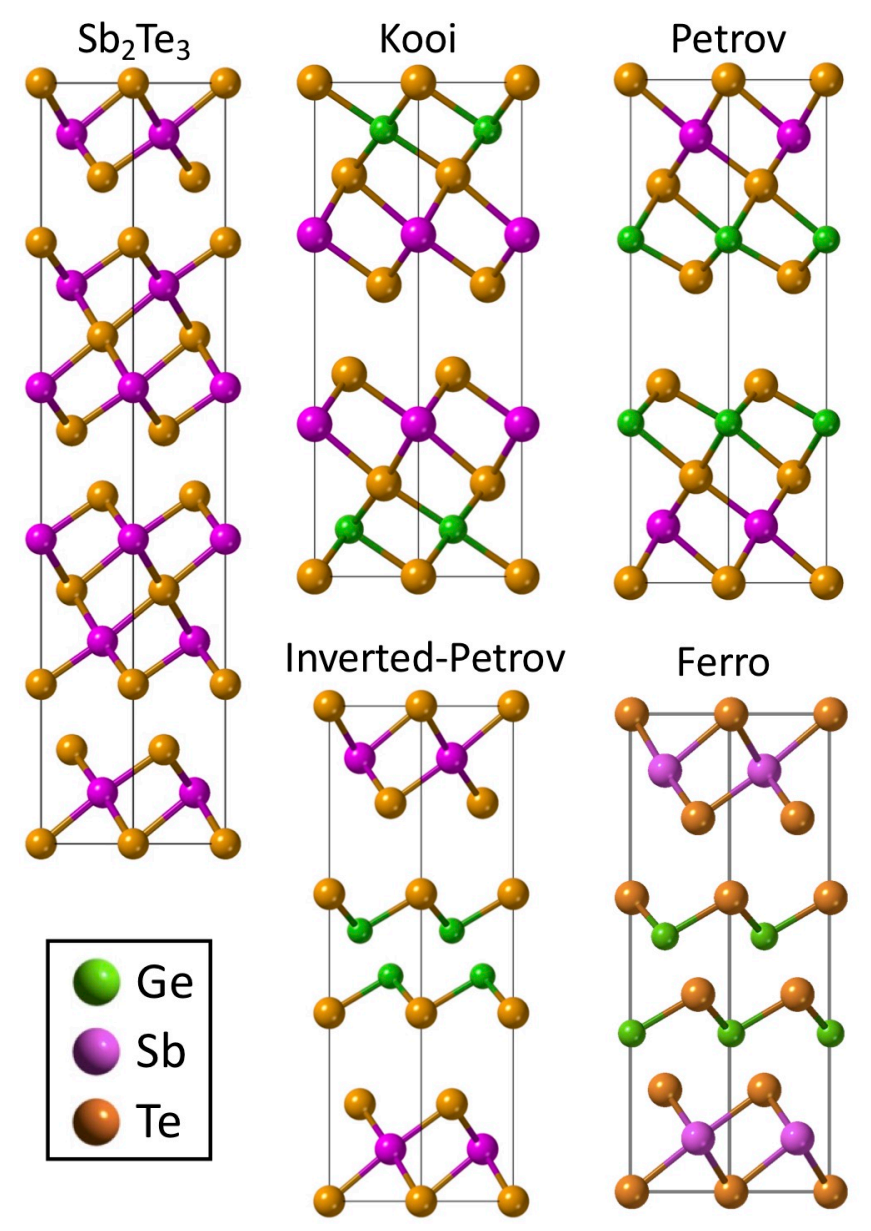

Figure 1. Crystal structures of $\mathrm{Sb}_{2} \mathrm{Te}_{3}$ and $(\mathrm{GeTe})_{2}\left(\mathrm{Sb}_{2} \mathrm{Te}_{3}\right)_{1}$ superlattices for Kooi, Petrov, Inverted-Petrov, and Ferro models. 

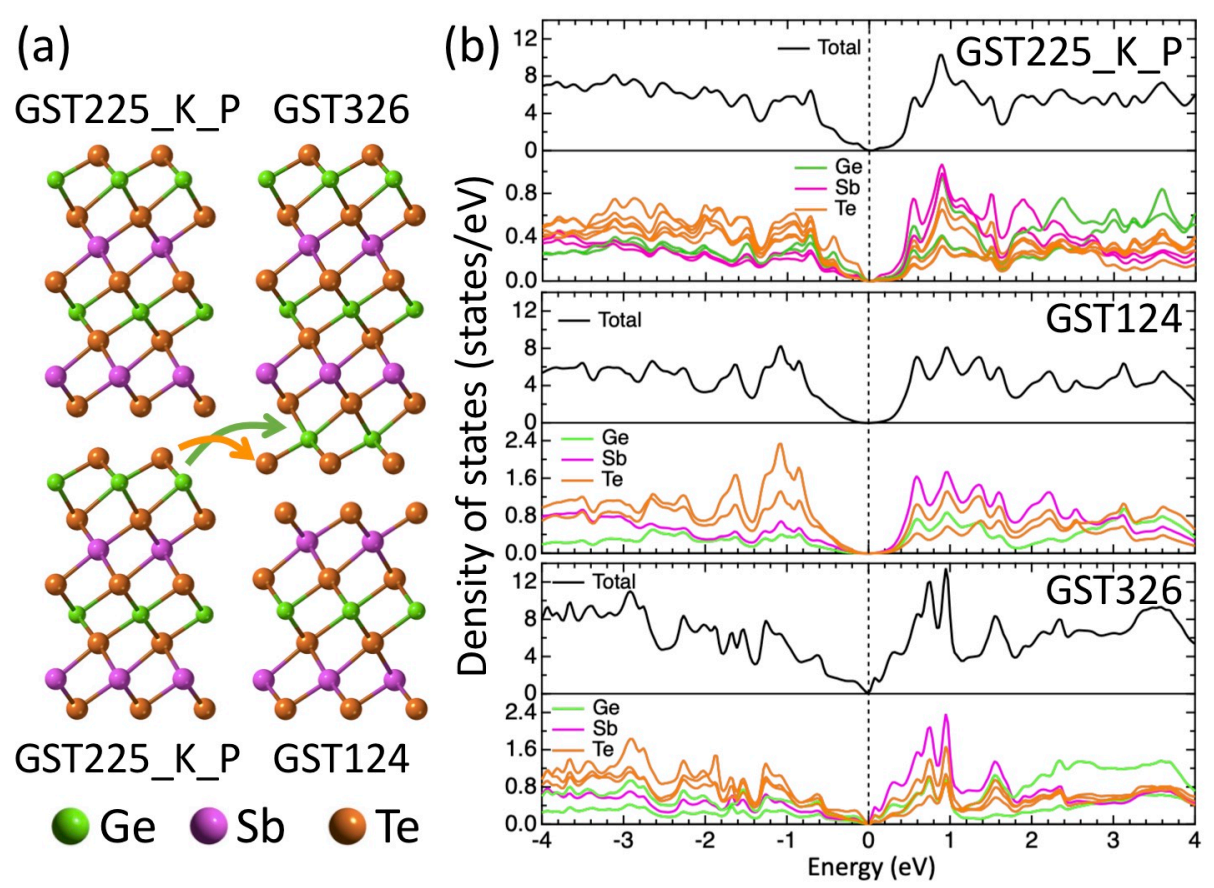

(c)
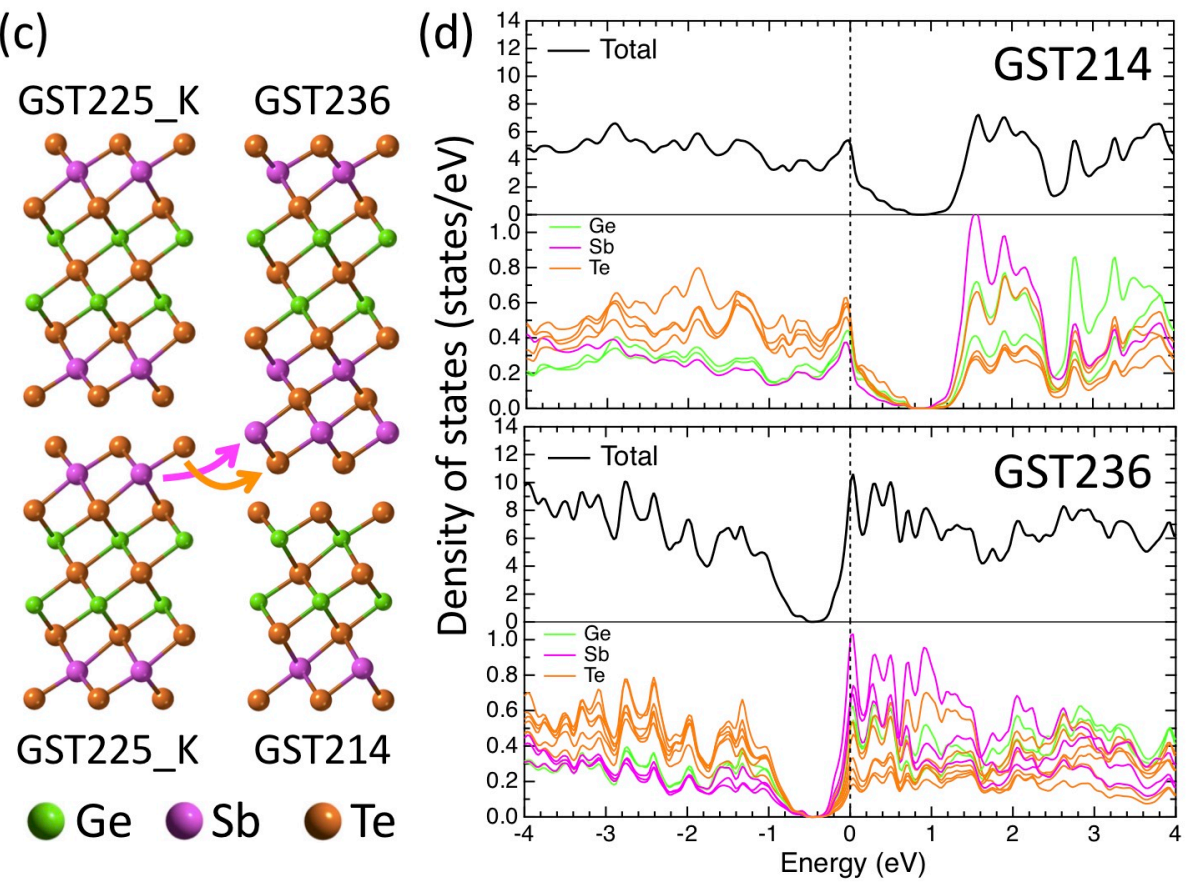

Figure 2. (a) Schematic GeTe bi-layer switching from two GST225_K_P blocks to GST124 and GST326 blocks. (b) The calculated electronic density of states for each block. (c) Schematic SbTe bi-layer switching from two GST225_K blocks to GST214 and GST236 blocks. (d) The calculated electronic density of states for GST214 and GST236 phases. The Fermi energy is shown at energy $0 \mathrm{eV}$. 


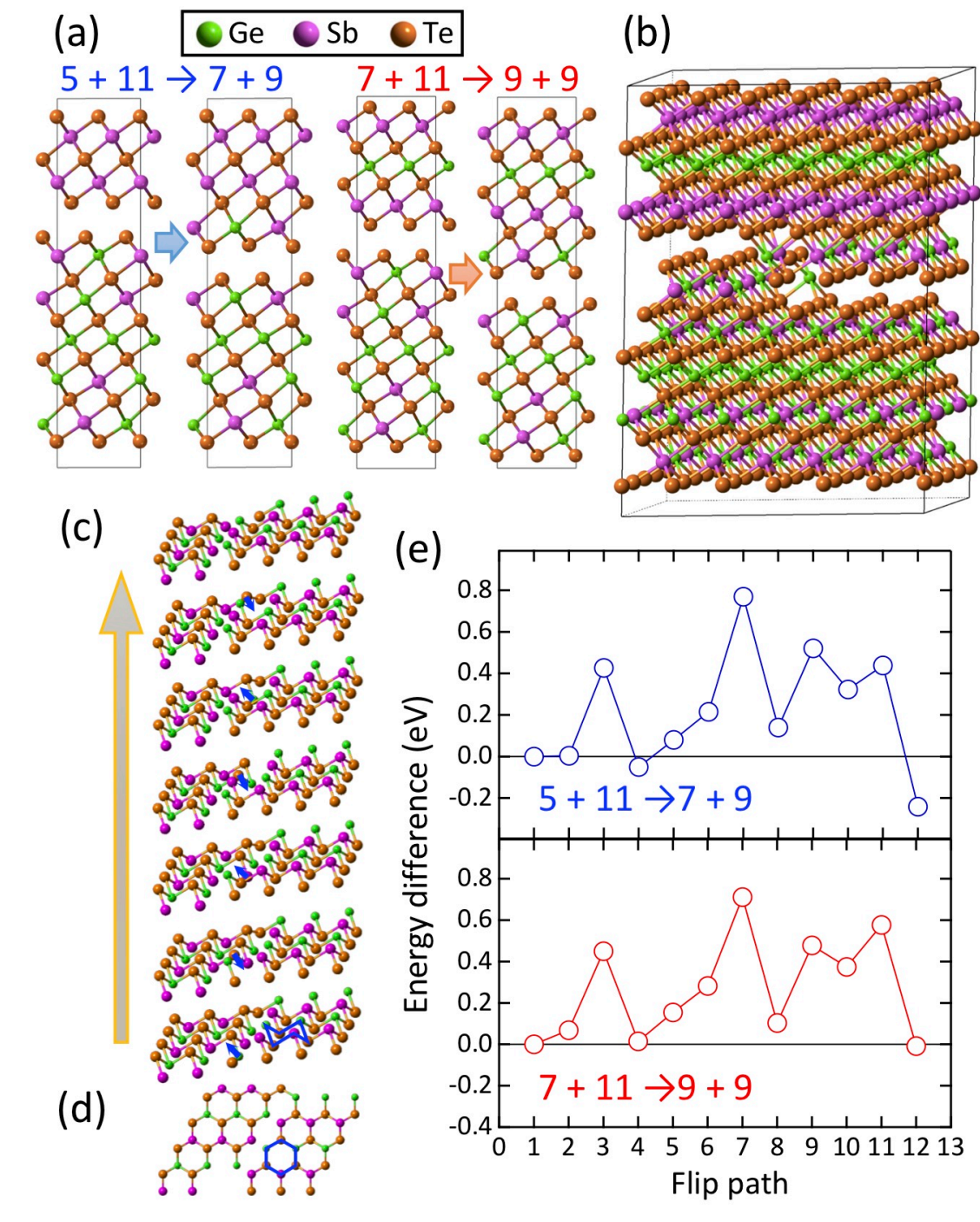

(b)

Figure 3. (a) Bi-layer switching of $5+11 \rightarrow 7+9$ and $7+11 \rightarrow 9+9$. (b) A snapshot during bi-layer switching. (c) Evolution of one-by-one atomic motion. (d) Top view of (c). (e) Calculated total energy difference for two switching processes. 


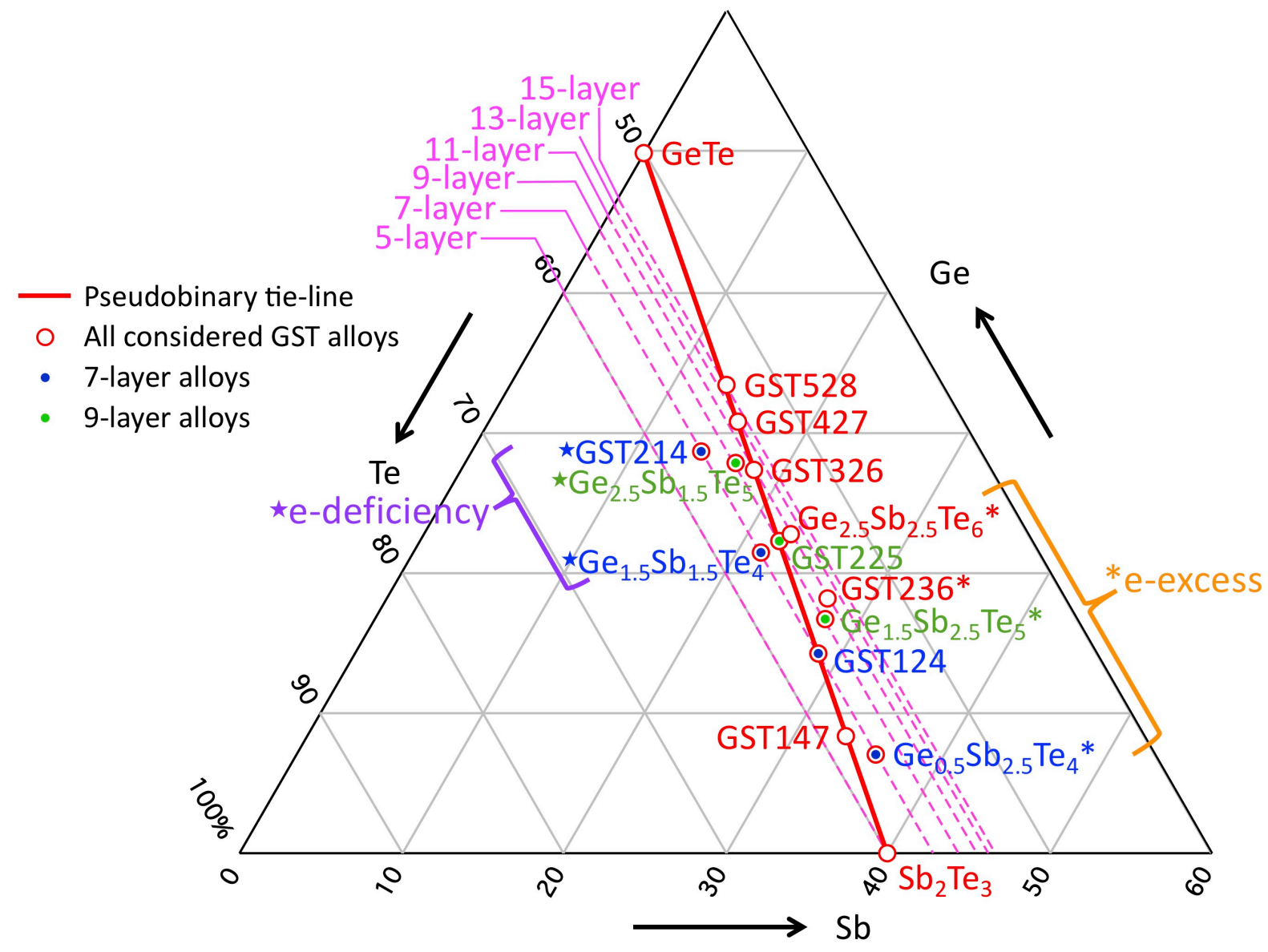

Figure 4. The Ge-Sb-Te ternary phase diagram showing a variety of ternary compounds including off-tie-line compositions. Depending on which side of the tie-line the composition is located, the region becomes either electron deficient or possesses a surplus of electrons. 
(a)

General crystalline GST

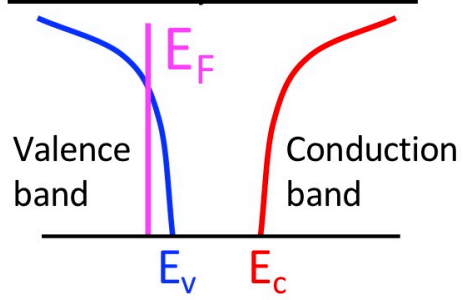

Hole doping

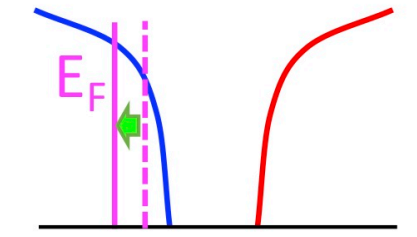

Electron doping

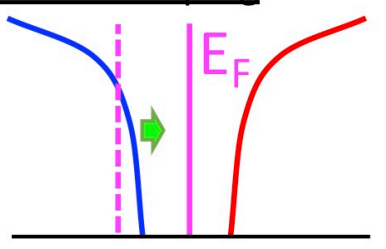

Energy

(c) SET (Long, low intensity pulse) = Annealing @moderate Temp.
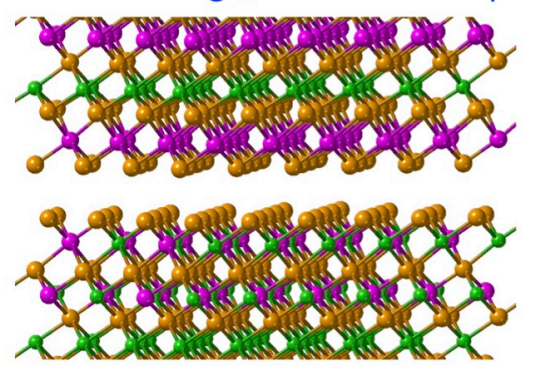

(b)
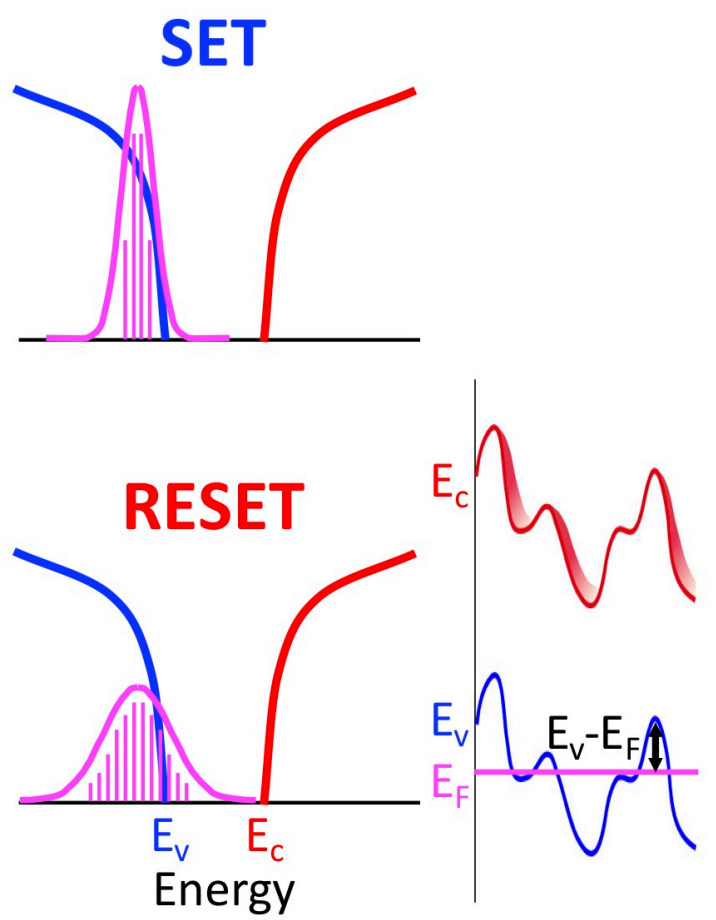

RESET (Short, high intensity pulse)

= Enough energy for reconfiguration

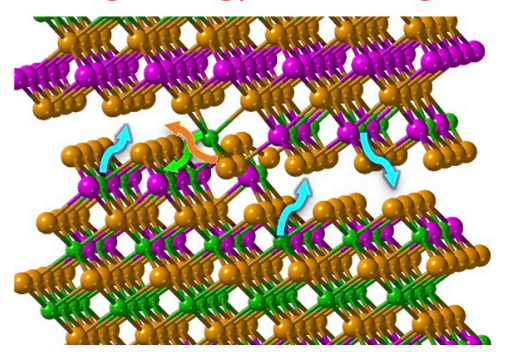

Figure 5. (a) DOS representing the relative positions of the Fermi energy depending on the composition. The dashed lines indicate the original position of the Fermi energy for general crystalline GST. (b) Schematic Fermi energy distribution for SET and RESET states and potential fluctuations for the RESET state. (c) Proposed models for low- (SET) and high(RESET) resistance states. 


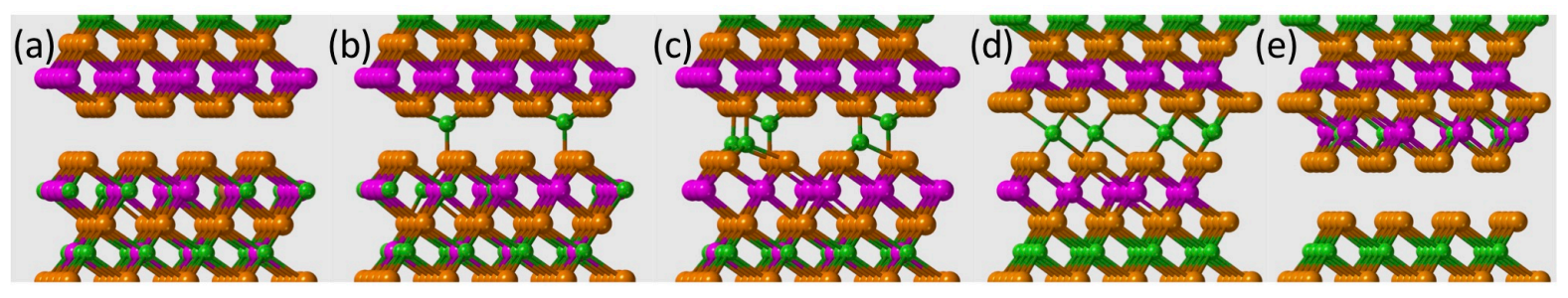

Figure 6. Schematic representation of bi-layer switching triggered by Ge atom switching. 